\title{
Option Pricing by Probability Distortion Operator Based on the Quantile Function
}

\author{
Luogen Yao $\mathbb{D}^{1,2}$ and Gang Yang $\mathbb{D}^{1,2}$ \\ ${ }^{1}$ School of Mathematics and Statistics, Hunan University of Commerce, Changsha 410205, China \\ ${ }^{2}$ Key Laboratory of Hunan Province for Mobile Business Intelligence, Hunan University of Commerce, Changsha 410205, China \\ Correspondence should be addressed to Gang Yang; yanggangmath@sina.com
}

Received 11 March 2019; Revised 5 May 2019; Accepted 15 May 2019; Published 16 June 2019

Academic Editor: Emilio Gómez-Déniz

Copyright (C) 2019 Luogen Yao and Gang Yang. This is an open access article distributed under the Creative Commons Attribution License, which permits unrestricted use, distribution, and reproduction in any medium, provided the original work is properly cited.

A new class of distortion operators based on quantile function is proposed for pricing options. It is shown that option prices obtained with our distortion operators are just the prices under mean correcting martingale measure in exponential Lévy models. In particular, Black-Scholes formula can be recuperated by our distortion operator. Simulation analysis shows that our distortion operator is superior to normal distortion operator and NIG distortion operator.

\section{Introduction}

With the convergence of financial and insurance markets, there has been a drastic increase in the trading and securitisation of insurance. The techniques adopted in insurance pricing are being considered for the option pricing. This has aroused wide interest in insurance pricing and how these techniques relate to financial theory.

In the actuarial literature, many probability transforms for pricing financial and insurance risks have been developed, such as the variance loading, the standard deviation loading, and the Esscher transform. Wang [1] and Wang [2] have proposed the following class of distortion operators based on normal distribution

$$
g_{\alpha}(u)=\Phi\left(\Phi^{-1}(u)+\alpha\right)
$$

where $\Phi$ denotes the standard normal cumulative distribution function (CDF) and $\alpha$ is a constant. The transform is now called the Wang transform, and the parameter $\alpha$ is considered to be a risk premium. The Wang transform not only possesses various desirable properties as a pricing method but also has a reasonable economic interpretation. However, Hamada and Sherris [3] have concluded that the Wang transform does not work well under non-Gaussian assumptions. Pelsser [4] also points out that, for general stochastic processes, Wang transform does not lead to a price which is consistent with the arbitrage free price.

It is well known that the returns of most financial assets have semiheavy tails and the actual kurtosis is higher than that of a normal distribution. The pricing principle approach of Wang must be somehow modified in order to capture the non-Gaussian feature of market prices. Many scholars have studied these issues from various perspectives. For example, Wang [2] proposes the two-parameter transformation

$$
g_{v, \lambda}(u)=T_{v}\left(\Phi^{-1}(u)+\lambda\right)
$$

and reports that the transform (2) is much better than (1) to fit the market data, where $T_{v}$ denotes the CDF of $t$-distribution with $\nu$ degree of freedom. Kijima and Muromachi [5] study the following transform:

$$
g_{v, \lambda}(u)=P_{v,-\lambda}\left[T_{v}^{-1}(u)\right]
$$

where $P_{\nu, \delta}$ denotes the CDF of noncentral $t$-distribution with $\nu$ degree of freedom and noncentrality parameter $\delta$. Kijima and Muromachi [6] consider the transform

$$
g_{\lambda}(u)=E\left[\Phi\left(G^{-1}(u)\right) Y+\lambda \mid Y\right]
$$


where $Y$ is a positive random variable and the expectation is taken with respect to $Y$. Here, $G(x)$ denotes the CDF of random variable $U / Y$ and $U$ represents a standard normal random variable, independent of $Y$. Godin et al. [7] introduce a new distortion based on Normal Inverse Gaussian (NIG) distribution

$$
g_{\alpha, \beta, \delta, \theta}(u)=\Phi^{N I G}\left(\Phi^{N I G^{-1}}(u)+\theta\right)
$$

where $\Phi^{N I G}$ denotes the NIG cumulative distribution function and show that the NIG-based distortion function performs better than the normal-based distortion function in Wang [1].

The price of an option obtained by any method should be arbitrage free. However, transforms (1)-(5) do not necessarily satisfy this property. We hold that we should make full use of the yield information to get the distortion functions. Inspired by the above works, we propose a new distortion operator based on the quantile function. It is shown that the celebrated Black-Scholes formula can be recuperated through our distortion operator. For a Lévy model, option price obtained with our distortion operator is just the price under mean correcting martingale measure (MCMM). Simulation analysis shows that our distortion operator is superior to normal distortion operator and NIG distortion operator.

\section{A New Class of Distortion Operators Based on Quantile Function}

Wang [1] recognizes that the Proportional Hazards probability transform cannot be applied consistently to assets and liabilities simultaneously. He proposes the so-called Wang transform aiming at integrating financial and actuarial insurance pricing theories. Let $F_{X}$ and $S_{X}$ be the distribution function and survival function of a risk $X$, respectively. Wang [1] defines the risk-adjusted premium for a risk $X$ by the Choquet integral representation

$$
\begin{aligned}
H[X ; \alpha]= & \int_{-\infty}^{0}\left\{g_{\alpha}\left[S_{X}(x)\right]-1\right\} d x \\
& +\int_{0}^{+\infty} g_{\alpha}\left[S_{X}(x)\right] d x,
\end{aligned}
$$

where $g_{\alpha}$ is a distortion function. When $X$ is a positive random variable, the Choquet integral representation has a simpler form

$$
H[X ; \alpha]=\int_{0}^{+\infty} g_{\alpha}\left[S_{X}(x)\right] d x
$$

In Wang transform, the tail probability distribution $S_{X}(x)$ is distorted by the function (1). One of the prominent properties of Wang transform is that it can recuperate the Black-Scholes formula in the standard Black-Scholes model.

The CDF of a real-valued random variable $X$ is the function given by $F_{X}(x)=P(X \leq x), x \in R$. It is well known that $F_{X}(x)$ is nondecreasing and right-continuous, which makes it a càdlàg function. Furthermore, $\lim _{x \rightarrow-\infty} F_{X}(x)=$
$0, \lim _{x \longrightarrow+\infty} F_{X}(x)=1$. If the CDF $F_{X}$ is strictly increasing, then $F_{X}^{-1}(y)$ is the unique real number $x$ such that $F_{X}(x)=y$. In such a case, this defines the inverse distribution function. However, the CDF does not, in general, have the ordinary inverse as it may not be strictly increasing.

Definition 1 (See [8]). For a nondecreasing function $h: \mathbf{R} \longrightarrow$ $\mathbf{R}$ with $h(-\infty)=\lim _{x \downarrow-\infty} h(x)$ and $h(\infty)=\lim _{x \uparrow \infty} h(x)$, the generalized inverse $h^{-}: R \longrightarrow \overline{\mathbf{R}}=[-\infty, \infty]$ of $h$ is defined by

$$
h^{-}(y)=\inf \{x \in \mathbf{R}: h(x) \geq y\}, \quad y \in \mathbf{R},
$$

with the convention that $\inf \{\phi\}=\infty$. If $h: \mathbf{R} \longrightarrow[0,1]$ is a $\mathrm{CDF}, h^{-}:[0,1] \longrightarrow \overline{\mathbf{R}}$ is also called the quantile function of $h$.

If $h$ is continuous and strictly increasing, $h^{-}$coincides with $h^{-1}$, the ordinary inverse of $h$ on $\operatorname{ran} h=\{h(x): x \in \mathbf{R}\}$, the range of $h$. If $h$ is continuous and nondecreasing, $h^{-}$is continuous, strictly increasing and

$$
\begin{gathered}
h(x)>y \Longleftrightarrow x>h^{-}(y), \\
h\left(h^{-}(y)\right)=y, \quad \forall y \in \operatorname{ran} h \cup\{\inf \operatorname{ran} h, \sup \operatorname{ran} h\} .
\end{gathered}
$$

The following theorem will be applied in this work.

Theorem 2. If $h$ is strictly increasing, then $\forall x \in \boldsymbol{R}, h^{-}(h(x-$ $0))=x$, where $h(x-0)$ is the left limit of $h$ at $x$.

Proof. $x \geq h^{-}(h(x-0))$ since $h(x) \geq h(x-0)$. On the other hand, $\forall \varepsilon>0$, there must exist some $x^{\prime} \in R$ such that

$$
x-\varepsilon<x^{\prime}<x .
$$

By the strictly monotonicity of $h$, we have

$$
h(x-\varepsilon)<h\left(x^{\prime}\right) \leq h(x-0) .
$$

This implies $h^{-}(h(x-0))=x$.

As $F_{X}$ may not be continuous in Theorem 2, we can only use the quantile function $F_{X}^{-}$, not the ordinary inverse $F_{X}^{-1}$. In this work, we will introduce a new class of distortion operators based on quantile function rather than on normal distribution.

Definition 3. Let $X$ be a random variable representing a financial (insurance) risk. We define the quantile distortion operator as

$$
g_{\lambda}^{X}(u)=F_{-X}\left(F_{-X}^{-}(u)+\lambda\right) .
$$

Note that unlike the distortion operators in (1)-(5), our distortion operator is completely determined by the distribution of the risk $X$. Essentially, our distortion operator shifts the $u^{\text {th }}$ quantile of $-X$ by a positive or negative value $\lambda$ and reevaluates the probability for the shifted quantile. We start our discussion with the following theorem to show the effect of the quantile distortion operator on the risk. 
Theorem 4. Let $X$ be a risk and $Z=h(X)$ be a transformation through a continuous, positive, and nondecreasing function $h$. If $F_{X}$ is strictly increasing, then we have

$$
H[Z ; X, \lambda] \triangleq \int g_{\lambda}^{X}\left(S_{Z}(x)\right) d x=E[h(X+\lambda)] .
$$

Proof. By definition, (9), and (10), we have

$$
\begin{aligned}
H[Z ; X, \lambda] & =\int g_{\lambda}^{X}\left(S_{Z}(x)\right) d x, \\
S_{Z}(x) & =P(Z>x)=P(h(X)>x) \\
& =P\left(X>h^{-}(x)\right)=P\left(-X<-h^{-}(x)\right) \\
& =F_{-X}\left(-h^{-}(x)-0\right) .
\end{aligned}
$$

As $F_{X}$ is strictly increasing and $F_{-X}(x)=1-F_{X}(-x-0)$, we can conclude that $F_{-X}$ is also strictly increasing. Due to Theorem 2, if we now apply the distortion $g_{\lambda}$ on $S_{Z}$, we have

$$
\begin{aligned}
g_{\lambda}^{X}\left(S_{Z}(x)\right) & =F_{-X}\left(F_{-X}^{-}\left(F_{-X}\left(-h^{-}(x)-0\right)\right)+\lambda\right) \\
& =F_{-X}\left(\lambda-h^{-}(x)\right) \\
& =P\left(-X \leq \lambda-h^{-}(x)\right) \\
& =P\left(X+\lambda \geq h^{-}(x)\right) \\
& =P(h(X+\lambda) \geq x),
\end{aligned}
$$

from which we conclude that

$$
\int g_{\lambda}^{X}\left(S_{Z}(x)\right) d x=E[h(X+\lambda)] .
$$

Remark 5. Under assumption that $X$ is a random variable with the $\operatorname{CDF} F_{X}$ whose probability density function is symmetric around 0, Hamada and Sherris [3] also derive the formula (14). In their proofs, the strict monotonically increasing property of the CDF is also adopted. However, Theorem 4 only needs the assumption that $F_{X}$ is strictly increasing. Hence, Theorem 4 generalizes the equivalent result found in Hamada and Sherris [3].

\section{Option Pricing}

Let us consider a market model where the stock price process $S=\left\{S_{t}, t \in[0, T]\right\}$ is a stochastic process of the following form:

$$
S_{t}=S_{0} e^{X_{t}}, \quad 0 \leq t \leq T,
$$

where $X=\left\{X_{t}, t \in[0, T]\right\}$ is a stochastic process defined on a stochastic basis $\left(\Omega, \mathscr{F}, \mathbf{F}=\left(\mathscr{F}_{t}\right)_{t \in[0, T]}, P\right)$ which satisfies the usual conditions. Moreover, in our market we have a riskless asset $B=\left\{B_{t}, t \in[0, T]\right\}$, evolving as

$$
B_{t}=\exp (r t),
$$

where $S_{0}>0, r>0$ are constants.
Under the assumption that the CDF $F_{X_{T}}$ of $X_{T}$ is strictly increasing, we now study how this distortion affects model (18) for asset prices and in particular if there is a value of $\lambda$ such that the discounted asset prices behave like risk-neutral asset prices. Note that $S_{T}$ can be expressed as $S_{T}=h\left(X_{T}\right)$, where $h(u)=S_{0} e^{u}$. Applying Theorem 4 , we have

$$
\begin{aligned}
H\left[S_{T} ; X_{T},-\lambda\right] & =E\left[h\left(X_{T}-\lambda\right)\right]=E\left[S_{0} e^{X_{T}-\lambda}\right] \\
& =S_{0} e^{-\lambda} E\left[e^{X_{T}}\right] .
\end{aligned}
$$

If we set

$$
\lambda^{*}=\ln E\left[e^{X_{T}}\right]-r T
$$

then the expression for $H\left[S_{T} ; X_{T},-\lambda\right]$ simplifies to

$$
H\left[S_{T} ; X_{T},-\lambda^{*}\right]=S_{0} e^{r T}
$$

In other words, under our distortion with a value of $\lambda^{*}$ given in (21), the price $S_{T}$ evolves like a risk-neutral asset.

Next, we will expound how our distortion operator is consistent with the option pricing theory. We need the following lemma.

Lemma 6. Let $X=\left\{X_{t}, t \in[0, T]\right\}$ be a P-Lévy process with the generating triplet $\left(\alpha, \sigma^{2}, \nu\right)_{P}$. One possible arbitrage free price of a European call option $C(T, K)=\left(S_{T}-K\right)^{+}$is given by

$$
C=E^{Q}\left[e^{-r T} C(T, K)\right],
$$

where $Q$ is the so-called MCMM under which $X=\left\{X_{t}, t \in\right.$ $[0, T]\}$ is a Lévy process with the generating triplet $(\alpha+r-$ $\left.\varphi(1), \sigma^{2}, \nu\right)_{\mathrm{Q}}$, and $\varphi$ is given by

$$
\begin{aligned}
\varphi(u) & \triangleq \ln E^{P}\left[e^{u X_{1}}\right] \\
& =u \alpha+\frac{1}{2} \sigma^{2}+\int\left(e^{u x}-1-x \mathbf{I}_{|x|<1}\right) \nu(d x) .
\end{aligned}
$$

Proof. We refer to Schoutens [9] for a proof of this result which is now standard in the literature.

In fact, (23) immediately yields the following formula:

$$
C=S_{0} e^{-T \varphi(1)} \int_{y}^{+\infty} e^{x} F_{X_{T}}(d x)-e^{-r T} K P \quad\left(X_{T}>y\right)
$$

where $y=(\varphi(1)-r) T-\ln \left(S_{0} / K\right)$.

Theorem 7. Let $X=\left\{X_{t}, t \in[0, T]\right\}$ be a P-Lévy process with the generating triplet $\left(\alpha, \sigma^{2}, \nu\right)_{P}$. If $F_{X_{T}}$ is strictly increasing, then we have

$$
e^{-r T} H\left[C(T, K) ; X_{T},-\lambda^{*}\right]=E^{Q}\left[e^{-r T} C(T, K)\right],
$$

where $\lambda^{*}=\ln E\left[e^{X_{T}}\right]-r T=(\varphi(1)-r) T$. Namely, option price under our distortion operator is just the price under MCMM. 
Proof. It is clear that $C(T, K)$ can be expressed as $C(T, K)=$ $h\left(X_{T}\right)$ for a function $h(u)=\left(S_{0} e^{u}-K\right)^{+}$. By Theorem 4 we have that

$$
\begin{aligned}
H & {\left[C(T, K) ; X_{T},-\lambda^{*}\right]=E\left[h\left(X_{T}-\lambda^{*}\right)\right] } \\
& =E\left[\left(S_{0} e^{X_{T}+(r-\varphi(1)) T}-K\right)^{+}\right] .
\end{aligned}
$$

Let $Y=\left\{Y_{t}, t \in[0, T]\right\}$, where $Y_{t}=X_{t}+(r-\varphi(1)) t$, and then

$$
H\left[C(T, K) ; X_{T},-\lambda^{*}\right]=E\left[\left(S_{0} e^{Y_{T}}-K\right)^{+}\right] .
$$

As $X=\left\{X_{t}, t \in[0, T]\right\}$ is a $P$-Lévy process with the generating triplet $\left(\alpha, \sigma^{2}, \nu\right)_{P}, Y=\left\{Y_{t}, t \in[0, T]\right\}$ is also a $P$-Lévy process, and the corresponding generating triplet is $\left(\alpha+r-\varphi(1), \sigma^{2}, v\right)_{P}$. Note that, by Lemma $6, X=\left\{X_{t}, t \in\right.$ $[0, T]\}$ is a $Q$ - Lévy process with the generating triplet $(\alpha+r-$ $\left.\varphi(1), \sigma^{2}, \nu\right)_{\mathrm{Q}}$. Thus, the CDF of $Y_{T}$ under probability measure $P$ is the same as the CDF of $X_{T}$ under probability measure $Q$. We then have

$$
\begin{aligned}
H & {\left[C(T, K) ; X_{T},-\lambda^{*}\right]=E^{Q}\left[\left(S_{0} e^{X_{T}}-K\right)^{+}\right] } \\
& =E^{Q}[C(T, K)] .
\end{aligned}
$$

In other words,

$$
e^{-r T} H\left[C(T, K) ; X_{T},-\lambda^{*}\right]=E^{Q}\left[e^{-r T} C(T, K)\right] .
$$

Theorem 7 implies that the price of a European call option which is evaluated with the pricing kernel associated with our distortion (13) with a parameter $\lambda^{*}$ is just this call price under MCMM Q. Therefore, our distortion operator is consistent with the option pricing theory.

Remark 8. Many popular market models based on Lévy processes in option pricing, such as Gaussian process, Normal Inverse Gaussian process, Meixner process, Generalized Hyperbolic process, Variance Gamma process, and CGMY process, can meet the condition under which the CDF of financial asset returns is strictly increasing. Thus, as for above Lévy processes, option price under our distortion operator is arbitrage free.

Remark 9. If $X$ is a NIG process, the NIG-distorted pricing kernel in (5) reduces to the Black-Scholes-type formula under the MCMM Q. As for other Lévy processes, we do not know whether the price under (5) is arbitrage free or not. Therefore, our distortion operator is more flexible.

Theorem 10. Let $X_{T}=\left(\mu-(1 / 2) \sigma^{2}\right) T+\sigma W_{T}$ and $Z=$ $h\left(X_{T}\right)$ be a transformation through a continuous, positive, and nondecreasing function $h$. We have

$$
\begin{aligned}
& F_{-X_{T}}\left(F_{-X_{T}}^{-}(P(Z>x))-\lambda^{*}\right) \\
& =\Phi\left(\Phi^{-1}(P(Z>x))-\alpha^{*}\right),
\end{aligned}
$$

where $\lambda^{*}=(\mu-r) T$ and $\alpha^{*}=(\mu-r) \sqrt{T} / \sigma$.
Proof. Note that

$$
\begin{aligned}
P(Z>x) & =P\left(h\left(X_{T}\right)>x\right)=P\left(X_{T}>h^{-}(x)\right) \\
& =1-P\left(X_{T} \leq h^{-}(x)\right) \\
& =1-\Phi\left(\frac{h^{-}(x)-\left(\mu-(1 / 2) \sigma^{2}\right) T}{\sigma \sqrt{T}}\right) \\
& =\Phi\left(\frac{\left(\mu-(1 / 2) \sigma^{2}\right) T-h^{-}(x)}{\sigma \sqrt{T}}\right) .
\end{aligned}
$$

So

$$
\begin{aligned}
\Phi & \left(\Phi^{-1}(P(Z>x))-\alpha^{*}\right) \\
& =\Phi\left(\frac{\left(\mu-(1 / 2) \sigma^{2}\right) T-h^{-}(x)}{\sigma \sqrt{T}}-\frac{(\mu-r) \sqrt{T}}{\sigma}\right) \\
& =\Phi\left(\frac{\left(r-(1 / 2) \sigma^{2}\right) T-h^{-}(x)}{\sigma \sqrt{T}}\right) .
\end{aligned}
$$

On the other hand,

$$
\begin{aligned}
& P(Z>x)=P\left(h\left(X_{T}\right)>x\right)=P\left(-X_{T}<-h^{-}(x)\right) \\
& \quad=F_{-X_{T}}\left(-h^{-}(x)\right), \\
& F_{-X_{T}}\left(F_{-X_{T}}^{-}(P(Z>x))-\lambda^{*}\right) \\
& \quad=F_{-X_{T}}\left(-h^{-}(x)-(\mu-r) T\right) \\
& =P\left(-X_{T} \leq-h^{-}(x)-(\mu-r) T\right) \\
& \quad=\Phi\left(\frac{\left(r-(1 / 2) \sigma^{2}\right) T-h^{-}(x)}{\sigma \sqrt{T}}\right) .
\end{aligned}
$$

Theorem 10 shows that the effect of our distortion operator with $\lambda^{*}=(\mu-r) T$ is the same as that of Wang's distortion operator with $\alpha^{*}=(\mu-r) \sqrt{T} / \sigma$ under Gaussian distribution assumptions.

Theorem 11. Let $X_{T}$ be a NIG distribution with parameters $(\alpha, \beta, \delta T, \mu T)$ and $Z=h\left(X_{T}\right)$ be a transformation through a continuous, positive, and nondecreasing function $h$. We have

$$
\begin{aligned}
F_{-X_{T}}\left(F_{-X_{T}}^{-}(P(Z>x))-\lambda^{*}\right) \\
=\Phi^{N I G}\left(\Phi^{N I G^{-1}}(P(Z>x))-\theta^{*}\right),
\end{aligned}
$$

where $\Phi^{N I G}$ is the cdf of NIG distribution with parameters $(\sqrt{\alpha \delta T},-\beta \sqrt{\delta T / \alpha}, \sqrt{\alpha \delta T}, 0)$, i.e., $\Phi^{N I G}(x)=N I G(x ; \sqrt{\alpha \delta T}$, $-\beta \sqrt{\delta T / \alpha}, \sqrt{\alpha \delta T}, 0)$ and 


$$
\begin{aligned}
& \lambda^{*}=\left(\mu-r-\delta\left(\sqrt{\alpha^{2}-(\beta+1)^{2}}-\sqrt{\alpha^{2}-\beta^{2}}\right)\right) T, \\
& \theta^{*}=\frac{\left(\mu-r-\delta\left(\sqrt{\alpha^{2}-(\beta+1)^{2}}-\sqrt{\alpha^{2}-\beta^{2}}\right)\right) \sqrt{T}}{\sqrt{\delta / \alpha}} .
\end{aligned}
$$

Proof. For $Z=h\left(X_{T}\right)$,

$$
\begin{aligned}
P(Z>x) & =P\left(h\left(X_{T}\right)>x\right)=1-P\left(X_{T} \leq h^{-}(x)\right) \\
& =1-P\left(\frac{X_{T}-\mu T}{\sqrt{\delta T / \alpha}} \leq \frac{h^{-}(x)-\mu T}{\sqrt{\delta T / \alpha}}\right) .
\end{aligned}
$$

As $\left(X_{T}-\mu T\right) / \sqrt{\delta T / \alpha}$ is a NIG distribution with parameters $(\sqrt{\alpha \delta T}, \beta \sqrt{\delta T / \alpha}, \sqrt{\alpha \delta T}, 0)$, we have

$$
\begin{aligned}
& P(Z>x) \\
& =1 \\
& \quad-N I G\left(\frac{h^{-}(x)-\mu T}{\sqrt{\delta T / \alpha}} ; \sqrt{\alpha \delta T}, \beta \sqrt{\frac{\delta T}{\alpha}}, \sqrt{\alpha \delta T}, 0\right) .
\end{aligned}
$$

Because of the symmetry property of the parameter $\beta$, we have

$$
\begin{aligned}
& P(Z>x) \\
& =N I G\left(-\frac{h^{-}(x)-\mu T}{\sqrt{\delta T / \alpha}} ; \sqrt{\alpha \delta T},-\beta \sqrt{\frac{\delta T}{\alpha}}, \sqrt{\alpha \delta T}, 0\right) \\
& =\Phi^{N I G}\left(\frac{\mu T-h^{-}(x)}{\sqrt{\delta T / \alpha}}\right) .
\end{aligned}
$$

So

$$
\begin{aligned}
& \Phi^{N I G}\left(\Phi^{N I G^{-1}}(P(Z>x))-\theta^{*}\right)=\Phi^{N I G}\left(\frac{\mu T-h^{-}(x)}{\sqrt{\delta T / \alpha}}-\theta^{*}\right) \\
& =\Phi^{N I G}\left(\frac{\left(r+\delta\left(\sqrt{\alpha^{2}-(\beta+1)^{2}}-\sqrt{\alpha^{2}-\beta^{2}}\right)\right) T-h^{-}(x)}{\sqrt{\delta T / \alpha}}\right) .
\end{aligned}
$$

On the other hand,

$$
\begin{aligned}
& F_{-X_{T}}\left(F_{-X_{T}}^{-}(P(Z>x))-\lambda^{*}\right) \\
& =F_{-X_{T}}\left(F_{-X_{T}}^{-}\left(F_{-X_{T}}\left(-h^{-}(x)\right)\right)-\lambda^{*}\right)=F_{-X_{T}}\left(-h^{-}(x)-\lambda^{*}\right) \\
& =1-P\left(\frac{X_{T}-\mu T}{\sqrt{\delta T / \alpha}} \leq \frac{h^{-}(x)+\lambda^{*}-\mu T}{\sqrt{\delta T / \alpha}}\right) \\
& =1-N I G\left(\frac{h^{-}(x)+\lambda^{*}-\mu T}{\sqrt{\frac{\delta T}{\alpha}}} ; \sqrt{\alpha \delta T}, \beta \sqrt{\frac{\delta T}{\alpha}}, \sqrt{\alpha \delta T}, 0\right) \\
& =N I G\left(-\frac{h^{-}(x)+\lambda^{*}-\mu T}{\sqrt{\delta T / \alpha}} ; \sqrt{\alpha \delta T},-\beta \sqrt{\frac{\delta T}{\alpha}}, \sqrt{\alpha \delta T}, 0\right) \\
& =\Phi^{N I G}\left(\frac{\left(r+\delta\left(\sqrt{\alpha^{2}-(\beta+1)^{2}}-\sqrt{\alpha^{2}-\beta^{2}}\right)\right) T-h^{-}(x)}{\sqrt{\delta T / \alpha}}\right) .
\end{aligned}
$$

Theorem 11 implies that the effect of our distortion operator is the same as that of NIG distortion operator under NIG distribution assumptions.

It is well known that Wang's distortion can recuperate the Black-Scholes formula in the standard Black-Scholes model. Now we show that our distortion can also recuperate the Black-Scholes formula.

In the standard Black-Scholes model, the stock prices follow a geometric Brownian motion with

$$
S_{t}=S_{0} \exp \left[\left(\mu-\frac{1}{2} \sigma^{2}\right) t+\sigma W_{t}\right], \quad 0 \leq t \leq T,
$$

where $W=\left\{W_{t}, 0 \leq t \leq T\right\}$ is a standard Brownian motion. Let $X_{T}=\left(\mu-(1 / 2) \sigma^{2}\right) T+\sigma W_{T}$, then $X_{T} \sim$ $N\left(\left(\mu-(1 / 2) \sigma^{2}\right) T, \sigma^{2} T\right)$, where $N\left(\mu, \sigma^{2}\right)$ denotes the normal distribution with mean $\mu$ and variance $\sigma^{2}$. By Theorem 4 , we have

$$
\begin{aligned}
& H(\left.C(T, K) ; X_{T},-\lambda^{*}\right)=E\left[h\left(X_{T}-\lambda^{*}\right)\right] \\
&=\int_{-\infty}^{+\infty}\left(S_{0} e^{x-\lambda^{*}}-K\right)^{+} \\
& \cdot \frac{1}{\sqrt{2 T \pi} \sigma} \exp \left(-\frac{\left(x-\left(\left(\mu-(1 / 2) \sigma^{2}\right) T\right)\right)^{2}}{2 \sigma^{2} T}\right) d x \\
&=\int_{-\infty}^{+\infty}\left(S_{0} e^{\sigma \sqrt{T} y+\left(\mu-(1 / 2) \sigma^{2}\right) T-\lambda^{*}}-K\right)^{+} \\
& \quad \cdot \frac{1}{\sqrt{2 \pi}} \exp \left(-\frac{y^{2}}{2}\right) d y .
\end{aligned}
$$

The values of $y$ for which $S_{0} e^{\sigma \sqrt{T} y+\left(\mu-(1 / 2) \sigma^{2}\right) T-\lambda^{*}} \geq K$ determine the region of integration. This region is $\left[y_{\min },+\infty\right)$ where

$$
y_{\min }=\frac{\ln \left(K / S_{0}\right)+\lambda^{*}-\left(\mu-(1 / 2) \sigma^{2}\right) T}{\sigma \sqrt{T}} .
$$

Hence

$$
\begin{aligned}
H( & \left.C(T, K) ; X_{T},-\lambda^{*}\right) \\
= & S_{0} e^{\mu T-\lambda^{*}} \int_{y_{\min }}^{+\infty} \frac{1}{\sqrt{2 \pi}} e^{-(1 / 2)(y-\sigma \sqrt{T})^{2}} d y \\
& -K\left(1-\Phi\left(y_{\min }\right)\right) \\
= & S_{0} e^{\mu T-\lambda^{*}}\left(1-\Phi\left(y_{\min }-\sigma \sqrt{T}\right)\right)-K \Phi\left(-y_{\min }\right) \\
= & S_{0} e^{\mu T-\lambda^{*}} \Phi\left(\sigma \sqrt{T}-y_{\min }\right)-K \Phi\left(-y_{\min }\right) .
\end{aligned}
$$

By (21),

$$
\lambda^{*}=\ln E\left[e^{X_{T}}\right]-r T=(\mu-r) T,
$$


from which we conclude that

$$
\begin{array}{rl}
e^{-r T} & H\left(C(T, K) ; X_{T},-\lambda^{*}\right) \\
= & S_{0} \Phi\left(\frac{\ln \left(S_{0} / K\right)+\left(r+(1 / 2) \sigma^{2}\right) T}{\sigma \sqrt{T}}\right) \\
& -e^{-r T} K \Phi\left(\frac{\ln \left(S_{0} / K\right)+\left(r-(1 / 2) \sigma^{2}\right) T}{\sigma \sqrt{T}}\right) .
\end{array}
$$

This demonstrates that our distortion operator (13) can also recuperate the Black-Scholes price of a European call option with $\lambda^{*}=(\mu-r) T$.

\section{Simulation Analysis}

On the basis of (13) under different types of data sets, we carry out an empirical analysis of the performance of our pricing kernel in this section. Our aim is twofold. First, we want to show that our distortion operator works well as Wang distortion operator when it is used in B-S model, and our distortion operator also works well as NIG distortion operator when it is used in NIG model. Our second aim is to show that our distortion operator performs better than Wang and NIG distortion operator in other Lévy option pricing models.

We use simulated data to test Wang, NIG, and our distortion operators, which provides a controlled setting for our analysis. These pricing kernels are tested on five different simulated data sets. Asset prices are simulated according to five well-known models for which theoretical option price formulae are available: geometric Brownian model, NIG model, Jump diffusion model, VG model, and Meixner model. In order to compare how the three distortions perform when evaluating option on underlying assets that do not have Gaussian log-returns, these pricing kernels were tested on these data sets. The valuation procedure of European call options is as follows.

Wang Distortion. (1) Simulate the stock price $S_{T}=S_{0} e^{X_{T}}$ and compute the empirical survival function $\widehat{S}_{S_{T}}$ of $S_{T}$;

(2) Using Wang distortion (1), the parameter $\widehat{\alpha}$ is then calibrated to verify the risk-neutral condition such that

$$
H\left(S_{T} ;-\widehat{\alpha}\right)=S_{0} e^{r T}
$$

(3) Using the simulated data, compute the empirical survival function $\widehat{S}_{C(T, K)}$ of European call option $C(T, K)=$ $\left(S_{T}-K\right)^{+}$

(4) $\widehat{\alpha}$ is used to compute the call option price

$$
e^{-r T} H(C(T, K) ;-\widehat{\alpha})=e^{-r T} \int_{0}^{+\infty} g_{\widehat{\alpha}}\left(\widehat{S}_{C(T, K)}\right) d x .
$$

NIG Distortion. (1) Simulate the stock price $S_{T}=S_{0} e^{X_{T}}$ and compute the empirical survival function $\widehat{S}_{S_{T}}$ and $\widehat{S}_{C(T, K)}$.

(2) Estimate the parameters $\alpha, \beta, \delta$ by maximum likelihood method from log-returns of simulated data.
(3) Using NIG distortion (5), the parameter $\widehat{\theta}$ is then calibrated to verify the risk-neutral condition such that

$$
H\left(S_{T} ;-\widehat{\theta}\right)=S_{0} e^{r T}
$$

(4) $\hat{\theta}$ is used to compute the call option price

$$
\begin{array}{rl}
e^{-r T} & H(C(T, K) ;-\widehat{\theta}) \\
& =e^{-r T} \int_{0}^{+\infty} g_{\alpha, \beta, \delta, \widehat{\theta}}\left(\widehat{S}_{C(T, K)}\right) d x .
\end{array}
$$

Our Distortion. (1) Simulate the stock price $S_{T}=S_{0} e^{X_{T}}$ and compute the empirical survival function $\widehat{S}_{S_{T}}$ and $\widehat{S}_{C(T, K)}$.

(2) Using our distortion (13), the parameter $\widehat{\lambda}$ is then calibrated to verify the risk-neutral condition such that

$$
H\left(S_{T} ; X_{T},-\hat{\lambda}\right)=S_{0} e^{r T}
$$

(3) $\hat{\lambda}$ is then used to compute the call option price

$$
\begin{array}{rl}
e^{-r T} & H\left(C(T, K) ; X_{T},-\widehat{\lambda}\right) \\
& =e^{-r T} \int_{0}^{+\infty} g_{\widehat{\lambda}}^{X_{T}}\left(\widehat{S}_{C(T, K)}\right) d x .
\end{array}
$$

4.1. Geometric Brownian Motion Model. In the standard Black-Scholes model, the stock prices follow a geometric Brownian motion with

$$
S_{t}=S_{0} \exp \left[\left(\mu-\frac{1}{2} \sigma^{2}\right) t+\sigma W_{t}\right], \quad 0 \leq t \leq T
$$

where $W=\left\{W_{t}, 0 \leq t \leq T\right\}$ is a standard Brownian motion. Note that the cdf of $X_{T}=\left(\mu-(1 / 2) \sigma^{2}\right) T+\sigma W_{T}$ is strictly increasing; option prices obtained with our distortion are just the Black-Scholes theoretical prices by Theorem 7 . In order to verify this, we simulate $3000 \log$-normal prices $S_{T}$ with drift $\mu=0.1$, volatility $\sigma=0.2$, and an initial price of $S_{0}=50$. The maturity is set at $T=0.5$; the risk free rate is set at $r=$ 0.05 . The strike prices $K$ range from 47 to 53 , which is used to calculated the option price.

The theoretical option prices are shown and compared with the option prices in Table 1, and the latter is obtained through procedure (49), (51), and (53). The prices obtained with Wang's distortion and our distortion are numerically equivalent to the Black-Scholes theoretical prices as expected. This first test shows that all three distortions perform well under Gaussian conditions.

4.2. Exponential NIG Model. The exponential NIG Lévy model for asset prices is of the form

$$
S_{t}=S_{0} e^{X_{t}}, \quad 0 \leq t \leq T,
$$


TABLE 1: Numerical European call option prices from a sample of asset prices simulated from B-S model.

\begin{tabular}{lccccccc}
\hline Strike price & 47 & 48 & 49 & 50 & 51 & 53 & 52 \\
Black-Scholes price & 5.2746 & 4.6111 & 4.0006 & 3.4444 & 2.9428 & 2.4950 & 2.0994 \\
Wang's price & 5.2727 & 4.6126 & 4.0057 & 3.4558 & 2.9554 & 2.5052 & 2.1057 \\
NIG price & 5.2710 & 4.6121 & 4.0066 & 3.4582 & 2.9593 & 2.5106 & 2.1125 \\
Our price & 5.2727 & 4.6126 & 4.0057 & 3.4558 & 2.9554 & 2.5052 \\
Wang's relative error & 0.0004 & 0.0003 & 0.0013 & 0.0033 & 0.0043 & 0.0041 & 0.0030 \\
NIG relative error & 0.0007 & 0.0002 & 0.0015 & 0.0040 & 0.0056 & 0.0062 & 0.0062 \\
Our relative error & 0.0004 & 0.0003 & 0.0013 & 0.0033 & 0.0043 & 0.0041 & 0.0030 \\
\hline
\end{tabular}

where $X=\left\{X_{t}, 0 \leq t \leq T\right\}$ is a NIG Lévy process with parameters $(\alpha, \beta, \delta, \mu)$; see Barndorff-Nielsen [10] and Rydberg [11]. The density function of $X_{1}$ is given by

$$
\begin{aligned}
& f_{\text {NIG }}(x ; \alpha, \beta, \delta, \mu) \\
& =\frac{\alpha \delta e^{\delta \gamma}}{\pi} \frac{K_{1}\left(\alpha \sqrt{\delta^{2}+(x-\mu)^{2}}\right)}{\sqrt{\delta^{2}+(x-\mu)^{2}}} e^{\beta(x-\mu)}, \quad x \in R,
\end{aligned}
$$

where $K_{\rho}$ is the modified Bessel function of the third kind with index $\rho$ given by

$$
K_{\rho}(x)=\frac{1}{2} \int_{0}^{+\infty} u^{\rho-1} \exp \left(-\frac{1}{2} x\left(u^{-1}+u\right)\right) d u,
$$

$$
x>0 \text {, }
$$

and $\gamma=\sqrt{\alpha^{2}-\beta^{2}}$. The parameter domain is $\delta>0, \alpha>$ $0, \alpha^{2}>\beta^{2}$, and $\mu \in R$.

As the cdf of $X_{T}$ is also strictly increasing, option prices with our distortion are just the prices under MCMM by Theorem 7. To verify this, we simulate 3000 stock prices $S_{T}$ with $\alpha=6, \beta=2, \mu=-0.5, \delta=1$, and an initial price of $S_{0}=20$. The risk free interest and the maturity of the call are, respectively, set at $r=0.08$ and $T=0.5$ years. The strike prices $K$ range from 17 to 23 , which is used to calculated the option price.

The theoretical option prices are shown and compared with the option prices in Table 2, and the latter is obtained through procedure (49), (51), and (53). The NIG price and our price are expected to be very accurate because of Godin et al. [7] and Theorem 7. The results of the simulation show that NIG distortion prices and our distortion prices overperform Wang's distortion prices for every strike price. The relative error for NIG prices and our prices remains smaller than $4 \%$ while it can climb up from $11 \%$ to $30 \%$ for Wang's distortion prices. The NIG distortion and our distortion have been proved to be more efficient in this case as well.

4.3. Jump Diffusion Model. In Merton [12], the prices of the asset are given by

$$
\begin{aligned}
S_{t} & =S_{0} \exp \left[\left(\mu-\frac{\sigma^{2}}{2}-\lambda\left(e^{\mu_{J}+\left(\sigma_{J}^{2} / 2\right)}-1\right)\right) t+\sigma W_{t}\right. \\
& \left.+\sum_{i=1}^{N_{t}} Y_{i}\right], \quad 0 \leq t \leq T,
\end{aligned}
$$

where $\mu \in R, \sigma>0, \lambda>0, \mu_{J} \in R, \sigma_{J}>0$ are constants; $W_{t}$ is a standard Brownian motion; $N_{t}$ is a Poisson process with intensity $\lambda$; and $\left\{Y_{i}\right\}_{i \geq 1}$ is a sequence of Gaussian random variables with mean $\mu_{J}$ and standard deviation $\sigma_{J}$. Variables $N_{t}, W_{t}$, and $Y_{i}$ are assumed to be all independent. Under MCMM, the price of a European call option with strike $K$ is given by Merton [12] as

$$
C_{T}=e^{-r T} \sum_{n=0}^{+\infty} e^{-\lambda T} \frac{(\lambda T)^{n}}{n !} C_{B S}\left(S_{n}, K, r, T, \sigma_{n}\right)
$$

where $\sigma_{n}^{2}=\sigma^{2}+n \sigma_{J}^{2} / T$,

$$
\begin{aligned}
S_{n}= & S_{0} \exp \left[n \mu_{J}+\frac{n}{2} \sigma_{J}^{2}-\lambda T\left(e^{\mu_{J}+0.5 \sigma_{J}^{2}}-1\right)\right], \\
C_{B S}(S, K, r, T, \sigma) & \\
= & S \Phi\left(\frac{\ln (S / K)+\left(r+(1 / 2) \sigma^{2}\right) T}{\sigma \sqrt{T}}\right) \\
& -e^{-r T} K \Phi\left(\frac{\ln (S / K)+\left(r-(1 / 2) \sigma^{2}\right) T}{\sigma \sqrt{T}}\right)
\end{aligned}
$$

is the value of a European call option with time to maturity $T$ and volatility $\sigma$ in a Black-Scholes model.

Once again, 3000 asset prices $S_{T}$ are simulated with $\mu=$ $0.25, \sigma=0.15, \lambda=1, \mu_{J}=0.2, \sigma_{J}=0.1$ and an initial price of $S_{0}=30$. The maturity and the risk free rate are, respectively, set at $T=0.5$ and $r=0.1$. The strike prices $K$ range from 27 to 33 , which is used to calculated the option price.

The theoretical option prices are shown and compared with the option prices in Table 3, and the latter is obtained through procedure (49), (51), and (53). Our distortion prices are expected to be very accurate because of Theorem 7 . The results of the simulation show that our distortion prices overperform Wang's distortion prices and NIG distortion prices for every strike price.

4.4. Exponential VG Model. Consider the following exponential VG stock price model:

$$
S_{t}=S_{0} e^{X_{t}}, \quad 0 \leq t \leq T,
$$


TABLE 2: Numerical European option prices from a sample of asset prices simulated from NIG model.

\begin{tabular}{lccccccc}
\hline Strike price & 17 & 18 & 19 & 20 & 21 & 22 & 23 \\
Price under MCMM & 5.4240 & 4.9783 & 4.5854 & 4.2383 & 3.9308 & 3.6575 & 3.4137 \\
Wang's price & 4.7464 & 4.2030 & 3.7277 & 3.3165 & 2.9603 & 2.6538 & 2.3884 \\
NIG price & 5.4267 & 4.9849 & 4.5935 & 4.2476 & 3.9401 & 3.6673 & 3.4233 \\
Our price & 5.4267 & 4.9849 & 4.5935 & 4.2476 & 3.9401 & 3.6673 & 3.4233 \\
Wang's relative error & 0.1422 & 0.1727 & 0.2008 & 0.2270 & 0.2504 & 0.2709 & 0.2906 \\
NIG relative error & 0.0005 & 0.0013 & 0.0018 & 0.0022 & 0.0024 & 0.0027 & 0.0028 \\
Our relative error & 0.0005 & 0.0013 & 0.0018 & 0.0022 & 0.0024 & 0.0027 & 0.0028 \\
\hline
\end{tabular}

TABLE 3: Numerical European call option prices from a sample of asset prices simulated from Merton model.

\begin{tabular}{lccccccc}
\hline Strike price & 27 & 28 & 29 & 30 & 31 & 32 & 33 \\
Price under MCMM & 4.7345 & 4.0541 & 3.4620 & 2.9553 & 2.5254 & 2.1611 & 1.8515 \\
Wang's price & 4.6560 & 3.9455 & 3.3164 & 2.7865 & 2.3465 & 1.9628 & 1.6437 \\
NIG price & 4.9895 & 4.2927 & 3.6724 & 3.1437 & 2.6974 & 2.3028 \\
Our price & 4.7573 & 4.0844 & 3.4906 & 2.9881 & 2.5645 & 2.1891 & 1.9683 \\
Wang' relative error & 0.0166 & 0.0268 & 0.0421 & 0.0571 & 0.0708 & 0.0918 & 0.1122 \\
NIG relative error & 0.0539 & 0.0589 & 0.0608 & 0.0638 & 0.0681 & 0.0656 & 0.0631 \\
Our relative error & 0.0048 & 0.0075 & 0.0082 & 0.0111 & 0.0155 & 0.0130 \\
\hline
\end{tabular}

TABLE 4: Numerical European call option prices from a sample of asset prices simulated from VG model.

\begin{tabular}{lccccccc}
\hline Strike price & 37 & 38 & 39 & 40 & 41 & 42 & 43 \\
Price under MCMM & 5.0259 & 4.2929 & 3.6154 & 2.9993 & 2.4487 & 1.9661 & 1.5516 \\
Wang's price & 4.9340 & 4.1888 & 3.5035 & 2.8855 & 2.3333 & 1.8527 & 1.4424 \\
NIG price & 4.9768 & 4.2366 & 3.5548 & 2.9384 & 2.3858 & 1.9028 \\
Our price & 4.9788 & 4.2395 & 3.5588 & 2.9432 & 2.3913 & 1.9086 & 1.49839 \\
Wang' relative error & 0.0183 & 0.0242 & 0.0310 & 0.0379 & 0.0471 & 0.0577 & 0.0704 \\
NIG relative error & 0.0098 & 0.0131 & 0.0168 & 0.0203 & 0.0257 & 0.0322 & 0.0408 \\
Our relative error & 0.0094 & 0.0124 & 0.0157 & 0.0187 & 0.0234 & 0.0293 & 0.0372 \\
\hline
\end{tabular}

where $X=\left\{X_{t}, 0 \leq t \leq T\right\}$ is a VG Lévy process with parameters $(\theta, \nu, \sigma, \mu)$. The density function of $X_{1}$ is given by

$$
\begin{aligned}
& f_{V G}(x ; \theta, \nu, \sigma, \mu) \\
& =\frac{2 e^{\theta(x-\mu) / \sigma^{2}}}{\nu^{1 / \nu} \sigma \Gamma(1 / \nu) \sqrt{2 \pi}}\left(\frac{|x-\mu|}{\sqrt{2 \sigma^{2} / \nu+\theta^{2}}}\right)^{1 / \nu-0.5} \\
& \cdot K_{1 / \nu-0.5}\left(\frac{|x-\mu| \sqrt{2 \sigma^{2} / \nu+\theta^{2}}}{\sigma^{2}}\right) .
\end{aligned}
$$

The parameter domain is $\nu>0, \sigma>0, \theta \in R, \mu \in R$.

We simulate 3000 stock prices $S_{T}$ with $\theta=-0.2, v=$ $0.1, \sigma=0.15, \mu=0.2$ and an initial price of $S_{0}=40$. The maturity and the risk free rate are set at $T=0.75$ and $r=0.05$, respectively. The option prices are calculated for the strike prices $K$ ranging from 37 to 43 .

In Table 4, the theoretical option prices are shown and compared with option prices obtained through procedures (49), (51), and (53), respectively. Once again, our distortion prices are very accurate because of Theorem 7 . The results of the simulation also show that our distortion prices overperform Wang's distortion prices and NIG distortion prices for every strike price.

4.5. Exponential Meixner Model. The last model on which all three distortions are tested is the exponential Meixner model. The prices of the asset are given by

$$
S_{t}=S_{0} e^{X_{t}}, \quad 0 \leq t \leq T,
$$

where $X=\left\{X_{t}, 0 \leq t \leq T\right\}$ is a Meixner Lévy process with parameters $(a, b, m, d)$. The density function of $X_{1}$ is given by

$$
\begin{gathered}
f_{M X}(x ; a, b, m, d)=\frac{(2 \cos (b / 2))^{2 d}}{2 a \pi \Gamma(2 d)} \\
\cdot \exp \left(\frac{\beta(x-m)}{a}\right)\left|\Gamma\left(d+\frac{i(x-m)}{a}\right)\right|^{2},
\end{gathered}
$$

where $x \in R, a>0,|b|<\pi, d>0, m \in R, i=\sqrt{-1}$.

Once more, 3000 asset prices $S_{T}$ are simulated with $a=$ $0.3, b=-0.1, m=0.15, d=0.2$ and an initial price of $S_{0}=50$. The maturity and the risk free rate are, respectively, 
TABLE 5: Numerical European call option prices from a sample of asset prices simulated from Meixner model.

\begin{tabular}{lccccccc}
\hline Strike price & 47 & 48 & 49 & 50 & 51 & 52 & 53 \\
Price under MCMM & 4.9900 & 4.0883 & 3.2148 & 2.3889 & 1.6504 & 1.0714 & 0.7010 \\
Wang's price & 5.1825 & 4.3165 & 3.4769 & 2.6839 & 1.9535 & 1.3087 & 0.7852 \\
NIG price & 4.9958 & 4.0922 & 3.2148 & 2.3951 & 1.6737 & 1.1094 & 0.7299 \\
Our price & 4.9951 & 4.0895 & 3.2086 & 2.3822 & 1.6524 & 1.0874 & 0.7184 \\
Wang' relative error & 0.0386 & 0.0558 & 0.0816 & 0.1235 & 0.1836 & 0.2215 & 0.1201 \\
NIG relative error & 0.0012 & 0.0010 & 0.0000 & 0.0026 & 0.0141 & 0.0355 \\
Our relative error & 0.0010 & 0.0003 & 0.0019 & 0.0028 & 0.0012 & 0.0150 & 0.0413 \\
\hline
\end{tabular}

set at $T=0.5$ and $r=0.08$. The option prices are calculated for the strike prices $K$ ranging from 47 to 53 .

In Table 5 the theoretical option prices are shown and compared with option prices obtained through procedures (49), (51), and (53), respectively. It can be seen that our distortion and the NIG distortion show an overall better performance than the normal distortion. We can also observe that, relatively speaking, our distortion performs better than the NIG distortion.

\section{Data Availability}

No data were used to support this study.

\section{Conflicts of Interest}

The authors declare that they have no conflicts of interest.

\section{Acknowledgments}

This work was supported by the Natural Science Foundation of Hunan Province, China (2019JJ40141; 2017JJ2127), and National Social Science Fund of China (15BJY122).

\section{References}

[1] S. S. Wang, "A class of distortion operators for pricing financial and insurance risks," Journal of Risk and Insurance, vol. 67, no. 1, pp. 15-36, 2000.

[2] S. S. Wang, "A universal framework for pricing financial and insurance risks," ASTIN Bulletin, vol. 32, no. 2, pp. 213-234, 2002.

[3] M. Hamada and M. Sherris, "Contingent claim pricing using probability distortion operators: Methods from insurance risk pricing and their relationship to financial theory," Applied Mathematical Finance, vol. 10, no. 1, pp. 19-47, 2003.

[4] A. Pelsser, "On the applicability of the Wang transform for pricing financial risks," The Journal of the International Actuarial Association, vol. 38, no. 1, pp. 171-181, 2008.

[5] M. Kijima and Y. Muromachi, "On the Wang transform with fat-tail distributions," in Proceedings of the Stanford-Tsukuba Workshop, Stanford University, 2006.

[6] M. Kijima and Y. Muromachi, "An extension of the Wang transform derived from Buhlmanni's economic premium principle for insurance risk," Insurance: Mathematics \& Economics, vol. 42, no. 3, pp. 887-896, 2008.
[7] F. Godin, S. Mayoral, and M. Morales, "Contingent Claim Pricing Using a Normal Inverse Gaussian Probability Distortion Operator," Journal of Risk and Insurance, vol. 79, no. 3, pp. 841866, 2012.

[8] P. Embrechts and M. Hofert, "A note on generalized inverses," Mathematical Methods of Operations Research, vol. 77, no. 3, pp. 423-432, 2013.

[9] W. Schoutens, Lévy Processes in Finance: Pricing Financial Derivatives, John Wiley and Sons, 2003.

[10] O. E. Barndorff-Nielsen, "Processes of normal inverse Gaussian type," Finance and Stochastics, vol. 2, no. 1, pp. 41-68, 1998.

[11] T. H. Rydberg, "The normal inverse Gaussian Lévy process: simulation and approximation," Communications in Statistics. Stochastic Models, vol. 13, no. 4, pp. 887-910, 1997.

[12] R. C. Merton, "Option pricing when underlying stock returns are discontinuous," Journal of Financial Economics, vol. 3, no. 1-2, pp. 125-144, 1976. 


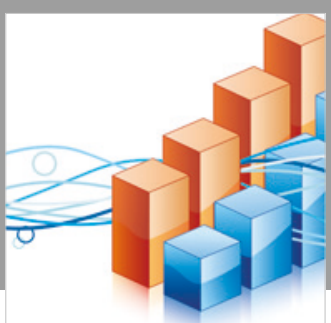

Advances in

Operations Research

\section{-n-m}
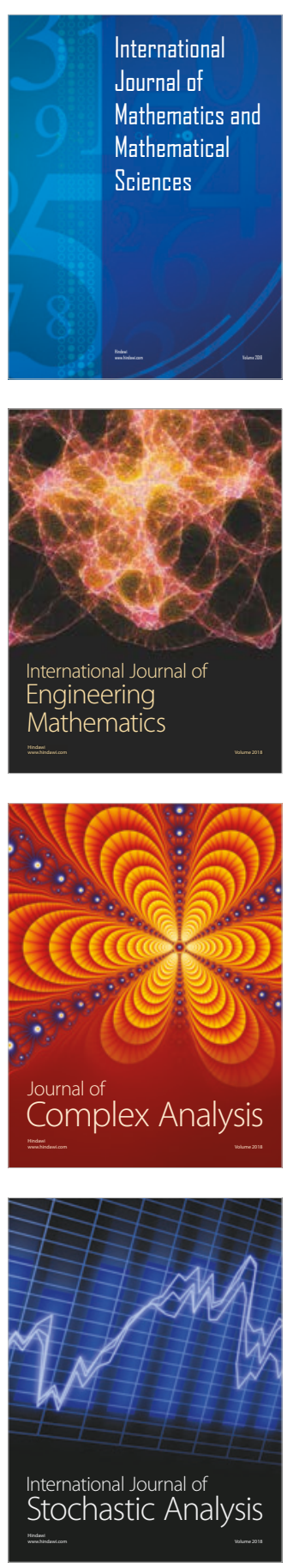
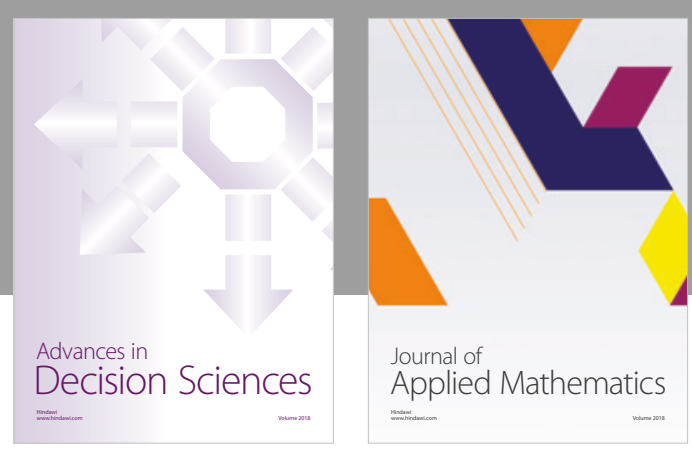

Journal of

Applied Mathematics
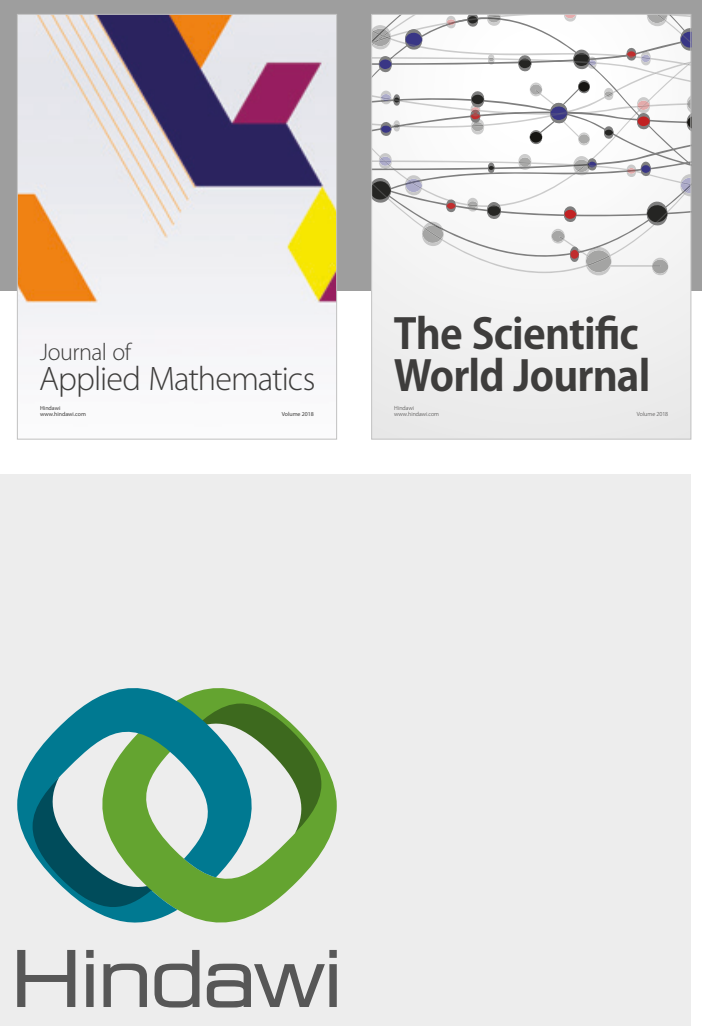

Submit your manuscripts at

www.hindawi.com

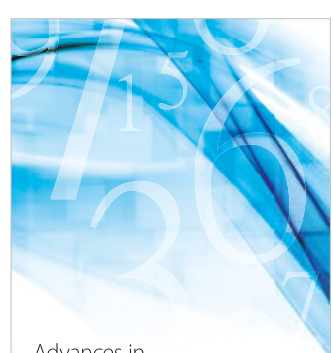

Advances in
Numerical Analysis
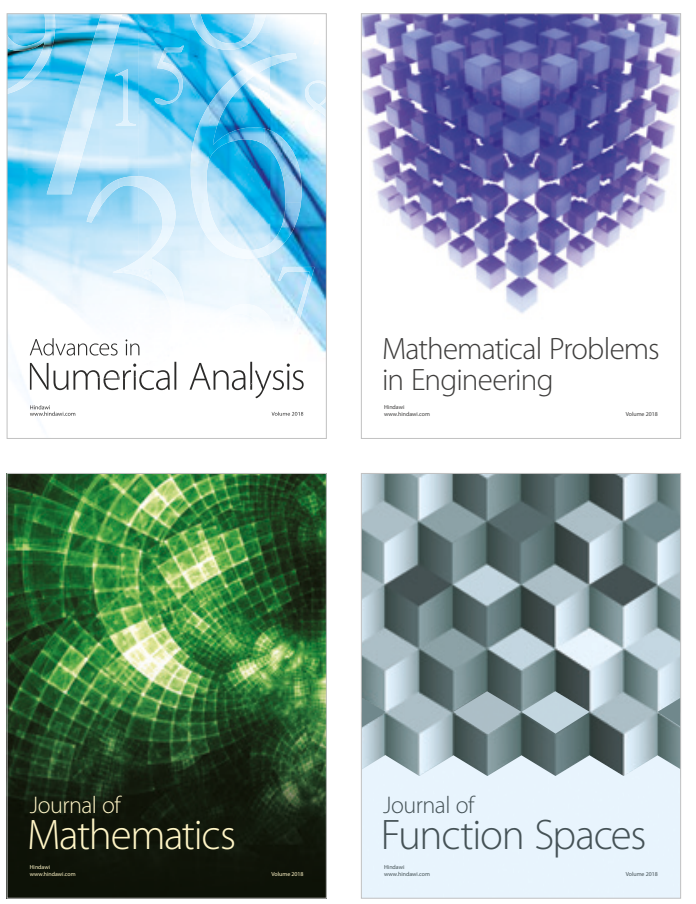

Mathematical Problems in Engineering

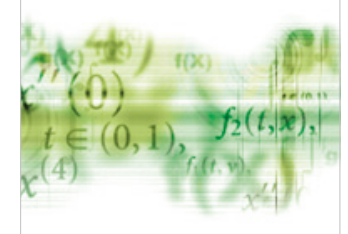

International Journal of

Differential Equations

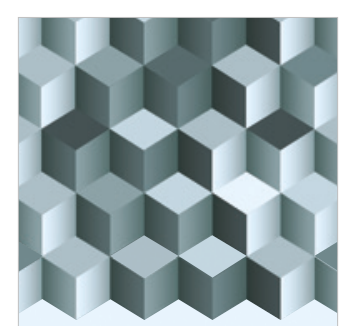

Journal of

Function Spaces

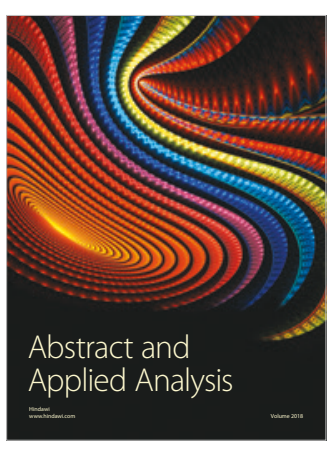

The Scientific

World Journal

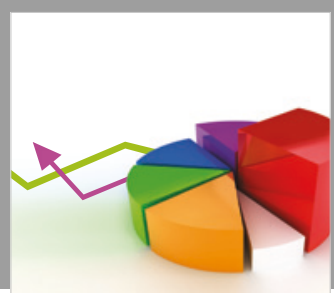

Journal of

Probability and Statistics
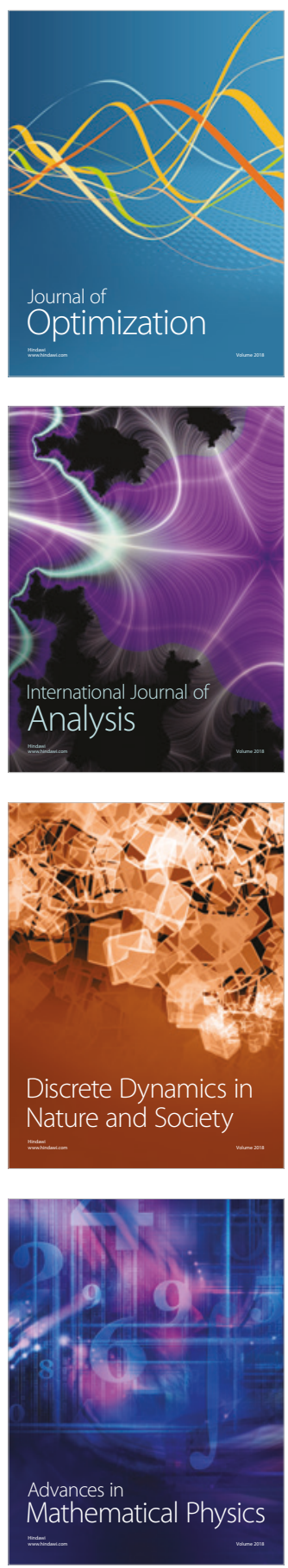